\title{
Medication use among participants in a clinical trial of massage for chronic neck pain. James Brent ${ }^{1}$, Matthew Bair ${ }^{2}$
}

${ }^{1}$ Indiana University School of Medicine, ${ }^{2}$ Indiana University of Medicine, Department of Internal Medicine

\section{Background and Hypothesis:}

Chronic neck pain is a common problem in veterans and is treated with NSAIDS and other analgesics. Massage is gaining traction due to patient demand, and concerns of long-term use of opioids and NSAIDS. We evaluated the use of analgesics and psychotropics among veterans in a clinical trial of massage for chronic neck pain. We hypothesized that analgesic use would be common and change over time.

\section{Project Methods:}

This is a retrospective chart review study of patients enrolled in the TOMCATT trial. The TOMCATT trial addressed biological, psychological, and social aspects to the treatment of chronic neck pain. Eligible patients were Veterans with chronic neck pain at the Richard L. Roudebush VA Medical Center in Indianapolis. The medications assessed by reviewing the pharmacy records embedded in the VA's EMR were acetaminophen, NSAIDS, muscle relaxants, opioids, topical analgesics, antidepressants, benzodiazepines, and anxiolytics. Patient medication use was assessed for 9 months during the trial.

\section{Results:}

Of the 235 veterans reviewed 33 were female and 202 were male. $11 \%$ of the patients were not prescribed medication during their trial. At kickoff date, $24 \%$ were prescribed acetaminophen, $29 \%$ NSAIDS, $40 \%$ muscle relaxants, $20 \%$ opioids, and $34 \%$ topical analgesics. At 9 months, those prescribed analgesics changed only slightly. Also, at kickoff date $35 \%$ were on antidepressants, $6 \%$ on benzodiazepines, and $5 \%$ on anxiolytics. These medications did not change much by 9 -months.

\section{Conclusion and Potential Impact}

Analgesics and antidepressants/anxiolytics were frequently prescribed among participants in this clinical trial. The most prescribed analgesics were muscle relaxants and topical analgesics. Overall, analgesic use did not change much over the course of the trial. In future work, we will monitor the continued use of meds to see if muscle relaxants and topicals remain the most used analgesics. 